TRANSACTIONS OF THE

AMERICAN MATHEMATICAL SOCIETY

Volume 326, Number 2, August 1991

\title{
LIE FLOWS OF CODIMENSION 3
}

\author{
E. GALLEGO AND A. REVENTÓS
}

\begin{abstract}
We study the following realization problem: given a Lie algebra of dimension 3 and an integer $q, 0 \leq q \leq 3$, is there a compact manifold endowed with a Lie flow transversely modeled on $\mathscr{G}$ and with structural Lie algebra of dimension $q$ ? We give here a quite complete answer to this problem but some questions remain still open (cf. §2).
\end{abstract}

\section{INTRODUCTION}

Among the class of foliations with a transverse structure, Lie foliations stand out. These are foliations transversely modeled on Lie groups. They have been studied by several authors, mainly by Fedida (cf. [3]). Apart from its intrinsic interest, the importance of this study is increased by the fact that they arise naturally in Molino's classification of Riemannian foliations [6].

To each Lie foliation are associated two Lie algebras, the Lie algebra $\mathscr{G}$ of the Lie group on which it is modeled and the structural Lie algebra $\mathscr{H}$. The latter algebra is the Lie algebra of the Lie foliation $\mathscr{F}$ restricted to the closure of any one of its leaves. In particular, it is a subalgebra of $\mathscr{G}$. We remark that although $\mathscr{H}$ is canonically associated to $\mathscr{F}, \mathscr{G}$ is not.

Thus, one natural and interesting question is to know which pairs of Lie algebras $(\mathscr{G}, \mathscr{H})$, with $\mathscr{H}$ a subalgebra of $\mathscr{G}$, can arise as transverse algebra and structural Lie algebra, respectively, of a Lie foliation $\mathscr{F}$ on a compact manifold $M$.

We shall study here a particular but interesting case; namely, given a Lie algebra of dimension 3 and an integer $q, 0 \leq q \leq 3$, is there a compact manifold endowed with a Lie flow transversely modeled on $\mathscr{G}$ and with structural Lie algebra of dimension $q$ ? For simplicity's sake we shall say that the pair $(\mathscr{G}, q)$ is (or is not) realizable.

By using the classification of the 3-dimensional Lie algebras and the fact that the structural Lie algebra of a Lie flow is abelian (cf. [1]) it becomes apparent that certain pairs $(\mathscr{G}, q)$ are not realizable (for instance, $(\mathrm{sl}(2), 2)$ and (so(3), 2) are not realizable because $\mathrm{sl}(2)$ and so(3) have no abelian subalgebras of dimension two).

Received by the editors April 14, 1989 and, in revised form, July 6, 1989.

1980 Mathematics Subject Classification (1985 Revision). Primary 53C12.

(c)1991 American Mathematical Society $0002-9947 / 91 \$ 1.00+\$ .25$ per page 
Nevertheless, in some cases the obstruction for certain pairs to be realizable is rooted in the compactness of $M$ and not based on purely algebraic reasons (for instance, the pair (affine, 0 ) is not realizable (cf. Theorem 1)).

We classify the 3-dimensional Lie algebras in 6 algebras $\mathscr{G}_{1}, \ldots, \mathscr{G}_{6}$ and two families $\mathscr{G}_{7}$ (parametrized by $k \in \mathbf{R}, k \neq 0$ ) and $\mathscr{G}_{8}$ (parametrized by $h \in \mathbf{R}$, $\left.h^{2}<4\right)$ (cf. §1). We obtain

Theorem 1. If the structural Lie algebra is zero, i.e. $\mathscr{F}$ is a compact foliation, then $\mathscr{G}_{1}, \mathscr{G}_{2}, \mathscr{G}_{3}$, and $\mathscr{G}_{4}$ are realizable. $\mathscr{G}_{5}$ and $\mathscr{G}_{6}$ are not realizable. $\mathscr{G}_{7}$ is realizable if and only if $k=-1$, and $\mathscr{G}_{8}$ is realizable if and only if $h=0$.

Theorem 2. If the structural Lie algebra has dimension 1, then $\mathscr{G}_{1}, \mathscr{G}_{2}, \mathscr{G}_{3}, \mathscr{G}_{4}$, and $\mathscr{G}_{5}$ are realizable. $\mathscr{G}_{6}$ and $\mathscr{G}_{7}$ are not realizable and $\mathscr{G}_{8}$ with $h=0$ is realizable.

We do not know any realization of $\mathscr{G}_{8}$ with $h \neq 0$ and 1-dimensional structural Lie algebra of dimension 1.

Finally, it is remarkable that the realization of the pair $\left(\mathscr{G}_{7}, 2\right)$ depends on $k$. In fact we have

Theorem 3. If the structural Lie algebra has dimension 2 , then $\mathscr{G}_{1}, \mathscr{G}_{5}$, and $\mathscr{G}_{8}$ with $h=0$ are realizable. $\mathscr{G}_{2}, \mathscr{G}_{3}, \mathscr{G}_{4}, \mathscr{G}_{6}$, and $\mathscr{G}_{7}$ with $k \in \mathbf{Q}$ are not realizable.

We give a realization of $\mathscr{G}_{7}$ with $k \notin \mathbf{Q}$. A characterization of those $k$ for which $\mathscr{G}_{7}$ is realizable and the $\mathscr{G}_{8}$ case, are still open.

We wish to thank Professors G. Hector and M. Nicolau for their helpful comments during the development of this work.

\section{Preliminary definitions and Results}

Let $\mathscr{F}$ be a smooth foliation of codimension $n$ on a smooth manifold $M$ given by an integrable subbundle $L \subset T M$. We denote by $\mathscr{L}(M, \mathscr{F})$ the Lie algebra of foliated vector fields, i.e. $X \in \mathscr{L}(M, \mathscr{F})$ if and only if $[X, Y] \in \Gamma L$ for all $Y \in \Gamma L$. Thus, the set of sections of $L, \Gamma L$, is an ideal of $\mathscr{L}(M, \mathscr{F})$. The elements of $\mathscr{L}(M, \mathscr{F}) / \Gamma L(M, \mathscr{F})$ are called basic vector fields.

If there is a family $\left\{X_{1}, \ldots, X_{n}\right\}$ of foliated vector fields of $M$ such that the corresponding family $\left\{\bar{X}_{1}, \ldots, \bar{X}_{n}\right\}$ of basic vector fields has rank $n$ everywhere, the foliation is called transversely parallelizable and $\left\{\bar{X}_{1}, \ldots, \bar{X}_{n}\right\}$ a transverse parallelism. If the vector subspace $\mathscr{G}$ of $\mathscr{X}(M, \mathscr{F})$ generated by $\left\{\bar{X}_{1}, \ldots, \bar{X}_{n}\right\}$ is a Lie subalgebra, the foliation is called a Lie foliation.

We shall use the following structure theorems (cf. [3] and [6]):

Theorem A. Let $\mathscr{F}$ be a transversally parallelizable foliation on a compact manifold $M$ of codimension $n$. Then:

(a) There is a Lie algebra $\mathscr{H}$ of dimension $q \leq n$.

(b) There is a locally trivial fibration $\pi: M \rightarrow W$ with compact fibre $F$ and $\operatorname{dim} W=n-q=m$. 
(c) There is a dense Lie $\mathscr{H}$-foliation on $F$ such that:

(i) The fibres of $\pi$ are the closures of the leaves of $\mathscr{F}$.

(ii) The foliation induced by $\mathscr{F}$ on each fibre of $\pi$ is isomorphic to the $\mathscr{H}$-foliation on $F$.

$\mathscr{H}$ is called the structural Lie algebra of $(M, \mathscr{F}), \pi$ the basic fibration, and $W$ the basic manifold. The foliation given by the fibres of $\pi$ is denoted by $\overline{\mathscr{F}}$. Note that $\operatorname{codim} \overline{\mathscr{F}}+q=\operatorname{codim} \mathscr{F}$.

Theorem B. Let $\mathscr{F}$ be a $\mathscr{G}$-foliation on a compact manifold $M$ and let $G$ be the connected simply-connected Lie group with Lie algebra $\mathscr{G}$. Let $p: \widetilde{M} \rightarrow M$ be the universal covering of $M$. Then there is a locally trivial fibration $D: \widetilde{M} \rightarrow$ $G$ equivariant by $\operatorname{Aut}(p)$ (i.e. if $D(x)=D(y)$ then $D(g x)=D(g y)$ for all $x, y \in \widetilde{M}$ and $g \in \operatorname{Aut}(p))$ such that the foliation $\widetilde{F}=p^{*} \mathscr{F}$ is given by the fibres of $D$.

The natural morphism $h: \pi_{1}(M) \rightarrow \operatorname{Diff}(G)$ is such that $\Gamma=\operatorname{im}(h) \subset G$, where the inclusion $G \subset \operatorname{Diff}(G)$ is by left translations.

We shall also use some cohomological properties of the foliation. Recall that the basic forms complex is given by the forms $\alpha \in \Omega^{*}(M)$ such that $\mathscr{L}_{X} \alpha=0$ and $i_{X} \alpha=0$ for all $X \in \Gamma L$. The cohomology of this complex, $H^{*}(M, \mathscr{F})$, is the basic cohomology of the foliated manifold $(M, \mathscr{F})$. If $H^{n}(M, \mathscr{F}) \neq 0$ we say that $\mathscr{F}$ is homologically orientable or unimodular. We have (cf. [5]):

Theorem C. Let $\mathscr{F}$ be an unimodular Lie $\mathscr{G}$-foliation on a compact manifold $M$. Then the Lie algebra $\mathscr{G}$ is unimodular.

Finally, we recall that the 3-dimensional Lie algebras can be classified in eight families.

$\mathscr{G}_{1}$ (Abelian):

$\mathscr{G}_{2}$ (Heisenberg):

$$
\left[e_{1}, e_{2}\right]=\left[e_{1}, e_{3}\right]=\left[e_{2}, e_{3}\right]=0 .
$$

$$
\left[e_{1}, e_{2}\right]=\left[e_{1}, e_{3}\right]=0, \quad\left[e_{2}, e_{3}\right]=e_{1} .
$$

$\mathscr{G}_{3}(\mathrm{so}(3)):$

$$
\left[e_{1}, e_{2}\right]=e_{3}, \quad\left[e_{2}, e_{3}\right]=e_{1}, \quad\left[e_{3}, e_{1}\right]=e_{2} .
$$

$\mathscr{G}_{4}(\mathrm{sl}(2)):$

$$
\left[e_{1}, e_{2}\right]=e_{3}, \quad\left[e_{2}, e_{3}\right]=-e_{1}, \quad\left[e_{3}, e_{1}\right]=e_{2} .
$$

$\mathscr{G}_{5}$ (Affine):

$\mathscr{G}_{6}:$

$$
\left[e_{1}, e_{2}\right]=e_{1}, \quad\left[e_{1}, e_{3}\right]=\left[e_{2}, e_{3}\right]=0 .
$$

$$
\left[e_{1}, e_{2}\right]=0, \quad\left[e_{1}, e_{3}\right]=e_{1}, \quad\left[e_{2}, e_{3}\right]=e_{1}+e_{2} .
$$

$\mathscr{G}_{7}:$

$$
\left[e_{1}, e_{2}\right]=0, \quad\left[e_{1}, e_{3}\right]=e_{1}, \quad\left[e_{2}, e_{3}\right]=k e_{2}, k \neq 0 \text {. }
$$


$\mathscr{G}_{8}:$

$$
\left[e_{1}, e_{2}\right]=0, \quad\left[e_{1}, e_{3}\right]=e_{2}, \quad\left[e_{2}, e_{3}\right]=-e_{1}+h e_{2}, h^{2}<4 .
$$

Notice that $\mathscr{G}_{1}, \mathscr{G}_{2}, \mathscr{G}_{3}$, and $\mathscr{G}_{4}$ are unimodular, $\mathscr{G}_{5}$ and $\mathscr{G}_{6}$ are not unimodular, $\mathscr{G}_{7}$ is unimodular only if $k=-1$, and $\mathscr{G}_{8}$ only if $h=0$.

Remark. We can think that $\mathscr{G}_{7}$ is parametrized by $k \in[-1,0) \cup(0,1]$. In fact two of these algebras are isomorphic if and only if $k \cdot k^{\prime}=1$.

\section{LIE FLOWS OF CODIMENSION 3}

Let $\mathscr{F}$ be a Lie flow of codimension 3 on a compact manifold $M$. Since the closures of the leaves of $\mathscr{F}$ are the fibres of a bundle (cf. Theorem A), there are four possible cases.

Case 1 . $\operatorname{codim} \overline{\mathscr{F}}=3$.

In this case $\mathscr{F}$ is compact and the basic bundle is $M \rightarrow M / \mathscr{F}$. Thus the basic cohomology coincides with the de Rham cohomology of the compact manifold $M / \mathscr{F}$, and hence $H^{3}(M / \mathscr{F}) \neq 0$. By Theorem $\mathrm{C}$, if such a flow exists it is transversely modeled on a unimodular Lie algebra. So $\mathscr{G}_{5}$ and $\mathscr{G}_{6}$ are not realizables, $\mathscr{G}_{7}$ is realizable (a priori) only if $k=-1$, and $\mathscr{G}_{8}$ only if $h=0$.

We now give examples for each one of the remainder algebras.

- $\mathscr{G}_{1}$ : Just consider the trivial bundle $T^{1} \times T^{3} \rightarrow T^{3}$.

- $\mathscr{G}_{2}$ : Consider the trivial bundle $T^{1} \times M \rightarrow M$ where $M$ is the homogeneous space $N / \Gamma$ of the Heisenberg group

$$
N=\left\{\left(\begin{array}{ccc}
1 & a & b \\
0 & 1 & c \\
0 & 0 & 1
\end{array}\right) ; a, b, c \in \mathbf{R}\right\}
$$

by the discret uniform subgroup $\Gamma$ of $N$ given by the matrices of $N$ with integer coefficients.

- $\mathscr{G}_{3}$ : Just consider the trivial bundle $T^{1} \times S^{3} \rightarrow S^{3}$.

- $\mathscr{G}_{4}$ : Consider the trivial bundle $T^{1} \times T_{1} W \rightarrow T_{1} W$ where $T_{1} W$ is the unit sphere bundle of the two hole torus $W . T_{1} W$ is the homogeneous space $\operatorname{PSL}(2, \mathbf{R}) / \pi_{1}(W)$ and therefore we have the desired example.

- $\mathscr{G}_{7}$ (with $k=-1$ ): Let $A \in \mathrm{SL}(2, \mathbf{Z})$ be a matrix with eigenvalues $\lambda$, $1 / \lambda$ (being $\lambda>0$ and $\lambda \neq 1$ ). We can give a solvable Lie group structure on $\mathbf{R}^{3}=\mathbf{R} \times \mathbf{R}^{2}$ by

$$
(t, u) \cdot(s, v)=\left(t+s, A^{t} \cdot v+u\right) .
$$

The Lie algebra of this group is $\mathscr{G}_{7}$ with $k=-1$ (cf. [4]). Moreover, the points of $\mathbf{R}^{3}$ with integer coordinates constitute a uniform discret subgroup $\Gamma$ of $\mathbf{R}^{3}$. The quotient is usually denoted by $T_{A}^{3}$. Then, one example of a Lie flow transversely modeled on $\mathscr{G}_{7}$, with $k=-1$, is given by the trivial bundle $T^{1} \times T_{A}^{3} \rightarrow T_{A}^{3}$. 
- $\mathscr{G}_{8}$ (with $\left.h=0\right)$ (P. Molino): Let us consider the flow given by the fibres of the trivial bundle $T^{1} \times T^{3} \rightarrow T^{3}$. Let $\theta^{0}, \theta^{1}, \theta^{2}, \theta^{3}$ denote the canonical coordinates in $T^{1} \times T^{3}$. The parallelism given by $\partial / \partial \theta^{1}, \partial / \partial \theta^{2}, \partial / \partial \theta^{3}$ makes the fibres of the bundle an abelian Lie foliation. But we have basic functions enough to modify this parallelism. In fact, we can take

$$
\begin{aligned}
& e_{1}=\cos \theta^{1} \cdot \partial / \partial \theta^{2}+\sin \theta^{1} \cdot \partial / \partial \theta^{3}, \\
& e_{2}=-\sin \theta^{1} \cdot \partial / \partial \theta^{2}+\cos \theta^{1} \cdot \partial / \partial \theta^{3}, \\
& e_{3}=-\partial / \partial \theta^{1},
\end{aligned}
$$

to obtain a new parallelism with $\left[e_{1}, e_{2}\right]=0,\left[e_{1}, e_{3}\right]=e_{2},\left[e_{2}, e_{3}\right]=-e_{1}$, i.e. the flow is also transversely modeled on $\mathscr{G}_{8}$ (with $h=0$ ).

Case 2. $\operatorname{codim} \overline{\mathscr{F}}=2$.

In this case we give examples for $\mathscr{G}_{1}, \mathscr{G}_{2}, \mathscr{G}_{3}, \mathscr{G}_{4}, \mathscr{G}_{5}$, and $\mathscr{G}_{8}$ (with $h=0$ ). We also prove that $\mathscr{G}_{6}$ and $\mathscr{G}_{7}$ are not realizable.

- $\mathscr{G}_{1}$ : One example is given by the flow $(X, 0)$ on $T^{2} \times T^{2}$ where $X$ is a dense linear flow on $T^{2}$.

- $\mathscr{G}_{2}$ : Let $M$ be the homogeneous space of the Heisenberg group considered before. The flow on $M \times T^{1}$ whose integral curves are given by

$$
\varphi_{t}(p)=\left(\left(\begin{array}{ccc}
1 & a & b+t \\
0 & 1 & c \\
0 & 0 & 1
\end{array}\right), t+d\right)
$$

where

$$
p=\left(\left(\begin{array}{ccc}
1 & a & b \\
0 & 1 & c \\
0 & 0 & 1
\end{array}\right), d\right) \quad \text { and } \quad d \in \mathbf{R} \backslash \mathbf{Q}
$$

is transverse to $M$, and the closure of each leaf is $T^{2}$. Hence it is one example of a $\mathscr{G}_{2}$-Lie flow with $\operatorname{codim} \overline{\mathscr{F}}=2$.

- $\mathscr{G}_{3}$ : As $S^{3}=\mathrm{SU}(3)$, an example can be constructed by suspending the representation $h: \pi_{1}\left(S^{1}\right) \rightarrow \operatorname{Diff}\left(S^{3}\right)$ given by $h(1)=\left(\begin{array}{cc}e^{i \alpha} & 0 \\ 0 & e^{i \alpha}\end{array}\right)$, where $\alpha \in$ $\mathbf{R} \backslash \mathbf{Q}$.

- $\mathscr{G}_{4}$ (A. ElKacimi): Let $\mathscr{F}_{0}$ be the transverse affine Lie flow on $T_{A}^{3}$ (cf. [1]). Using the fact that the affine group $G A$ can be considered, lifting the map

$$
\left(\begin{array}{ll}
a & b \\
0 & 1
\end{array}\right) \rightarrow \frac{1}{\sqrt{a}}\left(\begin{array}{ll}
a & b \\
0 & 1
\end{array}\right)
$$

of $G A$ in $\operatorname{SL}(2, \mathbf{R})$, as a Lie subgroup of $\widetilde{\mathrm{SL}}(2, \mathbf{R})$, and using also that the unfolding diagram of $\mathscr{F}_{0}$ (cf. Theorem B), $D_{0}: \widetilde{T}_{A}^{3} \rightarrow G A, \rho_{0}: \pi_{1}\left(T_{A}^{3}\right) \rightarrow G A$, the desired foliation can be constructed as follows:

Let $\widetilde{M}=\widetilde{T}_{A}^{3} \times \mathbf{R}$ be the universal covering of $M=T_{A}^{3} \times S^{1}$ and define $D: \widetilde{M} \rightarrow \widetilde{\mathrm{SL}}(2, \mathbf{R})$ and $\rho: \pi_{1}(M) \rightarrow \widetilde{\mathrm{SL}}(2, \mathbf{R})$ by $D(x, t)=D_{0} x \cdot \tilde{\varphi}(t)$ and 
$\rho^{\prime}(\gamma, n)=\rho_{0}(\gamma) \cdot \varphi(n)$, where $\tilde{\varphi}: \mathbf{R} \rightarrow \widetilde{\mathrm{SL}}(2, \mathbf{R})$ is a lift of the uniparametric subgroup $\varphi: \mathbf{R} \rightarrow \operatorname{SL}(2, \mathbf{R})$ given by

$$
\varphi=\left(\begin{array}{cc}
\cos t & \sin t \\
-\sin t & \cos t
\end{array}\right) .
$$

It turns out, using the fact that $\tilde{\varphi}(n)$ is in the center of $\widetilde{\mathrm{SL}}(2, \mathbf{R})$, that $\rho$ is an homomorphism and $D$ is equivariant (i.e., $D((\gamma, n) \cdot(x, t))=\rho(\gamma, n)$. $D(x, t))$. Thus the fibres of $D$ induce the desired Lie foliation on $M$ (cf. [2] for details).

- $\mathscr{G}_{5}$ : Let $X$ be the generator of the transversely affine Lie flow on $T_{A}^{3}$. As we have that $\mathscr{G}_{5}=\mathscr{A}+\mathbf{R}$, where $\mathscr{A}$ is the affine Lie algebra of dimension 2 , the vector field $(X, 0)$ on $T_{A}^{3} \times S^{1}$ is transversely modeled on $\mathscr{G}_{5}$ and $\operatorname{codim} \overline{\mathscr{F}}=2$.

- $\mathscr{G}_{6}$ and $\mathscr{G}_{7}$ are not realizable: Let $\mathscr{F}$ be a $\mathscr{G}_{6}$ or a $\mathscr{G}_{7}$ Lie flow on a compact manifold $M$. Fix a generator $X$ of $\mathscr{F}$ and a transverse parallelism $Y_{1}, Y_{2}, Y_{3}$ such that $\left[\bar{Y}_{1}, \bar{Y}_{2}\right]=0,\left[\bar{Y}_{1}, \bar{Y}_{3}\right]=\bar{Y}_{1},\left[\bar{Y}_{2}, \bar{Y}_{3}\right]=\bar{Y}_{1}+\bar{Y}_{2}$ for $\mathscr{G}_{6}$, and $\left[\bar{Y}_{1}, \bar{Y}_{2}\right]=0,\left[\bar{Y}_{1}, \bar{Y}_{3}\right]=\bar{Y}_{1},\left[\bar{Y}_{2}, \bar{Y}_{3}\right]=k Y_{2}$ for $\mathscr{G}_{7}$. Let $g$ be a Riemannian metric on $M$. Then we have the orthogonal decomposition $T M=T \overline{\mathscr{F}}+T \overline{\mathscr{F}}^{\perp}$ and we shall denote by $Z^{t}$ and $Z^{n}$ the tangent and the orthogonal parts of a vector field $Z$ on $M$.

The set $T=\left\{p \in M ; Y_{1}^{n}(p)=0\right\}$ is open. In fact, if $p \in T, Y_{1}$ is tangent to $\overline{\mathscr{F}}$ in $p$, therefore $Y_{2}^{n}, Y_{3}^{n}$ are independent in $p$. Hence they are independent in an open neighborhood $U$ of $p$ and we can write $Y_{1}^{n}=\lambda Y_{2}^{n}+\mu Y_{3}^{n}$ where $\lambda, \mu$ are basic functions on $U$. Computing now $\left[Y_{1}^{n}, Y_{2}^{n}\right]$ and $\left[Y_{1}^{n}, Y_{3}^{n}\right]$, we deduce the following system of differential equations:

$$
\begin{aligned}
Y_{2}^{n}(\lambda)+\mu \lambda+\mu & =0, \\
Y_{2}^{n}(\mu)+\mu^{2} & =0, \\
Y_{3}^{n}(\lambda)-\lambda^{2} & =0, \\
Y_{3}^{n}(\mu)-\mu \lambda+\mu & =0,
\end{aligned}
$$

for $\mathscr{G}_{6}$, and

$$
\begin{aligned}
Y_{2}^{n}(\lambda)+k \mu & =0, \\
Y_{2}^{n}(\mu) & =0, \\
Y_{3}^{n}(\lambda)+(1-k) \lambda & =0, \\
Y_{3}^{n}(\mu)+\mu & =0,
\end{aligned}
$$

for $\mathscr{G}_{7}$, with the initial conditions $\lambda(p)=\mu(p)=0$.

This implies that $\mu=0$ on the integral curves of $Y_{3}$ and $Y_{2}$. Due to transverse transitivity, $\mu=0$ on $U$. It follows, in a similar way, that $\lambda=0$ on $U$. Thus $Y=0$ on $U$ and $T$ is open.

As it is also closed and $M$ is supposed to be connected, $T=\varnothing$ or $T=M$. 
But if $T=M$, we arrive in both cases $\left(\mathscr{G}_{6}\right.$ and $\left.\mathscr{G}_{7}\right)$ to a contradiction. In fact, if we denote by $\theta^{0}, \theta^{1}, \theta^{2}, \theta^{3}$ the dual basis of $X, Y_{1}, Y_{2}, Y_{3}$ we have $d \theta^{2}=-\theta^{2} \wedge \theta^{3}$ in $\mathscr{G}_{6}$ and $d \theta^{2}=k \theta^{2} \wedge \theta^{3}(k \neq 0)$ in $\mathscr{G}_{7}$. As $\theta^{2}(Z)=$ $\theta^{3}(Z)=d \theta^{2}(Z, \cdot)=d \theta^{3}(Z, \cdot)=0$ for each vector field $Z$ tangent to $\bar{F}$, the 1 -forms $\theta^{2}$ and $\theta^{3}$ are projectable on the basic manifold $W=M / \bar{F}$.

So we would have an exact volume element on the compact manifold $W$, which is a contradiction.

Therefore $T=\varnothing$.

Next we consider the set $Q=\bigcup_{a \in \mathbf{R}} Q_{a}$ where $Q_{a}=\left\{p \in M ; Y_{2}^{n}(p)=\right.$ $\left.a Y_{1}^{n}(p)\right\}$.

$Q$ is open: If $p \in Q$, there is $a \in \mathbf{R}$ such that $Y_{2}^{n}(p)=a Y_{1}^{n}(p)$ and hence $Y_{3}^{n}$ and $Y_{1}^{n}$ are independent in $p$. So $Y_{2}^{n}=\lambda Y_{1}^{n}+\mu Y_{3}^{n}$ is an open neighborhood $U$ of $p$ with $\lambda(p)=a$ and $\mu(p)=0$. Computing now $\left[Y_{1}, Y_{2}^{n}\right]$, $\left[Y_{3}, Y_{2}^{n}\right]$ and considering their tangent and normal parts one obtains the equations:

$$
\begin{aligned}
Y_{1}(\lambda)+\mu & =0, \\
Y_{1}(\mu) & =0, \\
Y_{3}(\lambda)+1 & =0, \\
Y_{3}(\mu)-\mu & =0 .
\end{aligned}
$$

As before, this yields $\mu=0$, i.e. $Y_{2}^{n}=\lambda Y_{1}^{n}$ on $U$. Thus every point $x \in U$ is in $Q_{\lambda(x)} \subset Q$ and $Q$ is open.

$Q$ is closed: If $p \notin Q$, for each $a \in \mathbf{R}, Y_{2}^{n}(p) \neq a Y_{1}^{n}(p)$. In particular, $Y_{2}^{n}(p)=0$. As we have proved that $Y_{1}^{n} \neq 0$, the vector fields $Y_{1}, Y_{2}$ are linearly independent on $p$. Hence they are independent in an open neighborhood $U$ of $p$, i.e. $U \subset M \backslash Q$ and $Q$ is closed.

As $M$ is connected, $Q=\varnothing$ or $Q=M$.

If $Q=\varnothing, Y_{1}^{n}$ and $Y_{2}^{n}$ are linearly independent in each point. So there are differentiable functions $\lambda$ and $\mu$ globally defined on $M$, such that $Y_{3}^{n}=$ $\lambda Y_{1}^{n}+\mu Y_{2}^{n}$. Computing now $\left[Y_{1}, Y_{3}^{n}\right]$, we obtain $Y_{1}(\lambda)=1$, but as $M$ is compact this is impossible.

If $Q=M$, for each $p \in M$ there is $a(p) \in \mathbf{R}$ such that $Y_{2}^{n}(p)=a(p) Y_{1}^{n}(p)$. This gives rise to a differentiable basic function $a$ on $M$ with $Y_{2}^{n}=a \cdot Y_{1}^{n}$. Equivalently, $Y_{2}-a \cdot Y_{1}$ is everywhere tangent to $\overline{\mathscr{F}}$. Since $\left[Y_{3}, Y_{2}-a \cdot Y_{1}\right]$ must be in $\overline{\mathscr{F}}$ we obtain $Y_{3}(a)=-1$ for $\mathscr{G}_{6}$, which is again a contradiction, and $Y_{3}(a)=(1-k) a$ for $\mathscr{G}_{7}$. If $k \neq 1$, the only possibility is $a=0$ and so $Y_{2}$ is everywhere tangent to $\overline{\mathscr{F}}$. As before, this yields a contradiction because $d \theta^{1}=-\theta^{1} \wedge \theta^{3}$, with $\theta^{1}$ and $\theta^{3}$ projectables on $W=M / \bar{F}$. If $k=1$ it follows that $a$ is constant over the integral curves of $Y_{1}, Y_{2}, Y_{3}$, i.e. $a$ is constant. With $\omega^{0}, \omega^{1}, \omega^{2}, \omega^{3}$ the dual basis of $X, Y_{2}-a Y_{1}, Y_{1}, Y_{3}$, we obtain $d \omega^{2}=-\omega^{2} \wedge \omega^{3}$ with $\omega^{2}, \omega^{3}$ projectables on $W$, again a contradiction. This proves that $\mathscr{G}_{6}$ and $\mathscr{G}_{7}$ are not realizable. 
- $\mathscr{G}_{8}$ (with $h=0$ ): The same construction as before. If $\theta^{0}, \theta^{1}, \theta^{2}, \theta^{3}$ are the canonical coordinates on $T^{2} \times T^{2}$, the vector field $X=\partial / \partial \theta^{0}+\alpha \partial / \partial \theta^{1}$, $\alpha \in \mathbf{R} \backslash \mathbf{Q}$ is transversely abelian for the parallelism $\partial / \partial \theta^{1}, \partial / \partial \theta^{2}, \partial / \partial \theta^{3}$ and has $\operatorname{codim} \overline{\mathscr{F}}=2$. We modify this parallelism by taking

$$
\begin{aligned}
& e_{1}=\cos \theta^{2} \cdot \partial / \partial \theta^{1}+\sin \theta^{2} \cdot \partial / \partial \theta^{3}, \\
& e_{2}=-\sin \theta^{2} \cdot \partial / \partial \theta^{1}+\cos \theta^{2} \cdot \partial / \partial \theta^{3}, \\
& e_{3}=-\partial / \partial \theta^{2} .
\end{aligned}
$$

Thus $X$ is also transversely modeled on $\mathscr{G}_{8}$ (with $h=0$ ).

Case 3. $\operatorname{codim} \overline{\mathscr{F}}=1$.

In this case the structural Lie algebra has dimension 2. As this algebra is abelian (cf. [1]), $\mathscr{G}_{3}$ and $\mathscr{G}_{4}$ are not realizable because they do not have abelian subalgebras of dimension 2. Examples for the algebras $\mathscr{G}_{1}, \mathscr{G}_{2}, \mathscr{G}_{5}$, and $\mathscr{G}_{8}$ $(h=0)$ are given. For the algebra $\mathscr{G}_{7}$ we prove that the only realizable cases are when $k \notin \mathbf{Q}$, an example will be given. We also prove that $\mathscr{G}_{6}$ is not realizable.

- $\mathscr{G}_{1}$ : Consider the flow $(X, 0)$ on $T^{3} \times T^{1}$ where $X$ is a dense linear flow on $T^{3}$.

- $\mathscr{G}_{2}$ is not realizable: As $\mathscr{G}_{2}$ is unimodular and $\operatorname{codim} \overline{\mathscr{F}}=1, \mathscr{F}$ is unimodular (cf. [5]) and it follows, from the results by Molino (cf. [6]), that the central transverse sheaf $\mathscr{C}$ admits a global trivialization, i.e. there are independent foliated vector fields $v, w$ tangents to the $\mathscr{F}$ closure which commute, as transverse fields, with every global foliated vector field. In particular, $\left[v, e_{i}\right]=\left[w, e_{i}\right]=0$. Writing

$$
v=\lambda e_{1}+\mu e_{2}+\nu e_{3}, \quad w=\alpha e_{1}+\beta e_{2}+\gamma e_{3}
$$

we obtain $v=\lambda e_{1}$ and $w=\alpha e_{1}$, which is a contradiction.

- $\mathscr{G}_{5}$ : Let $X$ be the generator of the transversely affine Lie flow on $T_{A}^{3}$. The vector field $(X, \alpha \partial / \partial \theta)$ on $T_{A}^{3} \times S^{1}$, with $\alpha \in \mathbf{R} \backslash \mathbf{Q}$ and $\theta$ the coordinate function on $S^{1}$, is transversely modeled on $\mathscr{G}_{5}=\mathscr{A}+\mathbf{R}$ and $\operatorname{codim} \overline{\mathscr{F}}=1$.

- $\mathscr{G}_{8}(h=0)$ : The same construction as before. If $\theta^{0}, \theta^{1}, \theta^{2}, \theta^{3}$ are the canonical coordinates on $T^{3} \times T^{1}$, the vector field $X=\partial / \partial \theta^{0}+\alpha \partial / \partial \theta^{1}+$ $\beta \partial / \partial \theta^{2}$ with $\alpha, \beta$ rationally independent, admits

$$
\begin{aligned}
& e_{1}=\cos \theta^{3} \cdot \partial / \partial \theta^{0}+\sin \theta^{3} \cdot \partial / \partial \theta^{1}, \\
& e_{2}=-\sin \theta^{3} \cdot \partial / \partial \theta^{0}+\cos \theta^{3} \cdot \partial / \partial \theta^{1}, \\
& e_{3}=-\partial / \partial \theta^{3},
\end{aligned}
$$

as a transverse parallelism. But $e_{1}, e_{2}, e_{3}$ is a basis of $\mathscr{G}_{8}$ with $h=0$.

- Next we study the remainder algebras $\mathscr{G}_{6}, \mathscr{G}_{7}$, and $\mathscr{G}_{8}(h \neq 0)$. As the center of these algebras are trivial, the corresponding connected simply- 
connected groups $G_{6}, G_{7}, G_{8}$ can be obtained as $e^{t \cdot \text { ad } \alpha}, \alpha \in \mathscr{G}_{i}$ with $i=$ $1,2,3$. We find that these groups can be thought of as $\mathbf{R}^{3}=\mathbf{R}^{2} \times \mathbf{R}$ with the product $(p, t) \cdot\left(p^{\prime}, t^{\prime}\right)=\left(p+e^{-\Lambda t} \cdot p^{\prime}, t+t^{\prime}\right)$ and $\Lambda$ depending on the algebra.

For $\mathscr{G}_{6}$,

$$
\Lambda=\left(\begin{array}{ll}
1 & 1 \\
0 & 1
\end{array}\right), \quad e^{-\Lambda t}=\left(\begin{array}{cc}
e^{-t} & -t e^{-t} \\
0 & e^{-t}
\end{array}\right)
$$

For $\mathscr{G}_{7}$

$$
\Lambda=\left(\begin{array}{ll}
1 & 0 \\
0 & k
\end{array}\right), \quad e^{-\Lambda t}=\left(\begin{array}{cc}
e^{-t} & 0 \\
0 & e^{-k t}
\end{array}\right) .
$$

For $\mathscr{G}_{8}$,

$$
\Lambda=\left(\begin{array}{cc}
0 & 1 \\
-1 & h
\end{array}\right), \quad e^{-\Lambda t}=C(t) \cdot\left(\begin{array}{cc}
\cos (\varphi+t) & -\sin t \\
\sin t & \cos (\varphi-t)
\end{array}\right),
$$

where $C(t)=\frac{2}{\alpha} e^{\beta t}$ and $\alpha=\sqrt{4-h^{2}}, \beta=\tan \varphi=h / \alpha,(\sin \varphi=h / 2, \cos \varphi=$ $\alpha / 2)$.

The basis we have used to define the algebras are given in this case by the following left invariant fields.

For $\mathscr{G}_{6}$,

$$
e_{1}=e^{-t} \frac{\partial}{\partial x}, \quad e_{2}=-t e^{-t} \frac{\partial}{\partial x}+e^{-t} \frac{\partial}{\partial y}, \quad e_{3}=\frac{\partial}{\partial t} .
$$

For $\mathscr{G}_{7}$

$$
e_{1}=e^{-t} \frac{\partial}{\partial x}, \quad e_{2}=e^{-k t} \frac{\partial}{\partial y}, \quad e_{3}=\frac{\partial}{\partial t}
$$

For $\mathscr{G}_{8}$,

$$
\begin{aligned}
& e_{1}=\frac{2}{\alpha} e^{-\beta t}\left(\cos (t+\varphi) \frac{\partial}{\partial x}+\sin t \frac{\partial}{\partial y}\right), \\
& e_{2}=\frac{2}{\alpha} e^{-\beta t}\left(-\sin t \frac{\partial}{\partial x}+\cos (t-\varphi) \frac{\partial}{\partial y}\right), \\
& e_{3}=-\frac{\alpha}{2} \frac{\partial}{\partial t} .
\end{aligned}
$$

Suppose now that we have a $\operatorname{codim} \overline{\mathscr{F}}=1$ realization on a compact manifold $M$ of one of these algebras. We shall denote the algebra by $\mathscr{G}$ and the corresponding group by $G$. The basic fibration is: $T^{3} \rightarrow M \rightarrow T^{1}$ and, as $\pi_{1}\left(T^{3}\right)=\mathbf{Z}^{3}, \pi_{1}\left(T^{1}\right)=\mathbf{Z}$, and $\pi_{2}\left(T^{1}\right)=0$, the corresponding homotopy exact sequence is $0 \rightarrow \mathbf{Z}^{3} \rightarrow \pi_{1}(M) \rightarrow \mathbf{Z} \rightarrow 0$.

Since this exact sequence has a section, $\pi_{1}(M)$ is the semidirect product of $\mathbf{Z}^{3}$ with $\mathbf{Z}$, i.e. $\pi_{1}(M)$ is the product $\mathbf{Z}^{3} \times \mathbf{Z}$ with the operation $(x, t) \cdot(y, x)=$ $(x+t \cdot y, t+s)$ where $t \cdot y$ represents the natural action of $\mathbf{Z}$ on $\mathbf{Z}^{3}$. To be precise, if $\varphi: T^{3} \rightarrow T^{3}$ is the diffeomorphism which gives the bundle, then the action is $t \cdot y=\varphi_{*}^{t} \cdot y$ where $\varphi_{*}: \pi_{1}\left(T^{3}\right) \rightarrow \pi_{1}\left(T^{3}\right)$ is the morphism induced by $\varphi$. We shall denote the group by $\mathbf{Z}^{3} \times{ }_{\varphi} \mathbf{Z}$. 
Since $\mathscr{F}$ is a Lie foliation, we have the unfolding diagram:

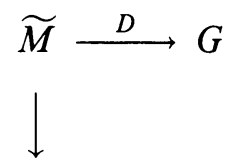

$M$

and the holonomy representation $h: \pi_{1}(M) \rightarrow h\left(\pi_{1}(M)\right)=\Gamma \subset G$ with $D(\gamma \cdot \tilde{x})=h(\gamma) \cdot D \tilde{x}, \tilde{x} \in \widetilde{M}, \gamma \in \pi_{1}(M)$.

As $M / \bar{F}$ is diffeomorphic to $G / \bar{\Gamma}$ (cf., for instance, [5]) we have that $\bar{\Gamma}$ is a two-dimensional closed subgroup of $G$.

The Lie algebra $\mathscr{H}$ of $\bar{\Gamma}_{e}$ (the identity component of $\bar{\Gamma}$ ) is named the structural Lie algebra of $\mathscr{F}$ and, in the case of flows, it is abelian (cf. [1]).

But it is easy to see that the only two-dimensional abelian subalgebra of $\mathscr{G}$ is $\left\langle e_{1}, e_{2}\right\rangle$, thus $\mathscr{H}=\left\langle e_{1}, e_{2}\right\rangle$. Looking at the expressions for $e_{1}$ and $e_{2}$ in $\mathscr{G}_{6}, \mathscr{G}_{7}$, and $\mathscr{G}_{8}$, we see that $\mathscr{H}=\langle\partial / \partial x, \partial / \partial y\rangle$ and hence $\bar{\Gamma} \simeq \mathbf{R}^{2} \times \mathbf{Z} \varepsilon$, $\varepsilon>0$.

Notice that $\bar{\Gamma}_{e}=\mathbf{R}^{2} \times\{0\}$ is abelian.

Lemma. Let $A$ be an abelian subgroup of $\Gamma$. Then $A$ is contained in $\mathbf{R}^{2} \times\{0\}$ or there is an element $a=\left(a_{1}, a_{2}, a_{3}\right)$ with $a_{3} \neq 0$ such that $A=\left\{a^{n}, n \in \mathbf{Z}\right\}$. Proof. If $A$ is not in $\mathbf{R}^{2} \times\{0\}$, then $A \cap\left(\mathbf{R}^{2} \times\{0\}\right)=0 \in \mathbf{R}^{3}$.

Otherwise, there is $(p, 0) \in A, p \neq 0$, and $(q, t) \in A, t \neq 0$. As $A$ is abelian we have that $(p, 0)(q, t)=(q, t)(p, 0)$. Then $q+e^{-\Lambda t} \cdot p=q+p$ and this implies that $t=0$, except for $\mathscr{G}_{8}$ with $h=0$, but this case is not considered here. Therefore $A \cap\left(\mathbf{R}^{2} \times\{0\}\right)=0 \in \mathbf{R}^{3}$.

In particular, $A$ has at most one element in each level $\mathbf{R}^{2} \times\{m \varepsilon\}, m \in \mathbf{Z}$. In fact, $a_{1} \cdot a_{2}^{-1} \in A \cap\left(\mathbf{R}^{2} \times\{0\}\right)=0$ and $a_{1}=a_{2}$.

Let $a=\left(a_{1}, a_{2}, n \varepsilon\right)$ be the element of $A$ in the lower level. For each $b=\left(b_{1}, b_{2}, m \varepsilon\right) \in A$, we put $m=n d+r$; then $b a^{-d}$ is an element of $A$ in the $r \varepsilon$ level and hence $r=0$, i.e. $b=a^{d}$ and this proves the lemma.

Proposition 1. Let the notation be as above. Then $\left(\mathbf{R}^{2} \times\{0\}\right) \cap \Gamma=h\left(\mathbf{Z}^{3}\right)$.

Proof. Applying the lemma we have four possibilities:

(i) $h\left(\mathbf{Z}^{3}\right)$ and $h(\mathbf{Z})$ are both contained in $\mathbf{R}^{2} \times\{0\}$. Then $\Gamma$, generated by $h\left(\mathbf{Z}^{3}\right)$ and $h(\mathbf{Z})$, is contained in $\mathbf{R}^{2} \times\{0\}$, which contradicts $\mathbf{R}^{3} / \bar{\Gamma}=S^{1}$.

(ii) $h\left(\mathbf{Z}^{3}\right)$ is contained in $\mathbf{R}^{2} \times\{0\}$ and $h(\mathbf{Z})=\left\{a^{n}, n \in \mathbf{Z}\right\}$ with $a \notin$ $\mathbf{R}^{2} \times\{0\}$. As $h\left(\mathbf{Z}^{3}\right)$ is a normal subgroup of $\Gamma$, for each $b \in h\left(\mathbf{Z}^{3}\right)$ we have $a b a^{-1}=b^{\prime}$ which is in $h\left(\mathbf{Z}^{3}\right)$. Hence, the elements of $\left(\mathbf{R}^{2} \times\{0\}\right) \cap \Gamma$ can be written as

$$
\sigma=b_{1} a^{r_{1}} b_{2} a^{r_{2}} b_{3} a^{r_{3}} \cdots b_{k} a^{r_{k}}
$$

with $\sum r_{i}=0$ and $b_{i} \in h\left(\mathbf{Z}^{3}\right)$. That is, $\sigma=\tilde{b} \cdot a^{\sum r_{i}}=\tilde{b} \in h\left(\mathbf{Z}^{3}\right)$, i.e. $\left(\mathbf{R}^{2} \times\{0\}\right) \cap \Gamma=h\left(\mathbf{Z}^{3}\right)$. 
(iii) $h(\mathbf{Z})$ is contained in $\mathbf{R}^{2} \times\{0\}$ and $h\left(\mathbf{Z}^{3}\right)=\left\{a^{n}, n \in \mathbf{Z}\right\}$ with $a \notin$ $\mathbf{R}^{2} \times\{0\}$. In this case, $\Gamma$ is abelian because if we let $h(1)=b$ we have $b a b^{-1}=a^{k}$. This implies $k=1$ and $a b=b a$. As in (ii), this implies that $\Gamma \cap \mathbf{R}^{2} \times\{0\}=h(\mathbf{Z})$ which is not dense in $\mathbf{R}^{2} \times\{0\}$.

(iv) $h(\mathbf{Z})=\left\{a^{n}, n \in \mathbf{Z}\right\}$ and $h\left(\mathbf{Z}^{3}\right)=\left\{b^{n}, n \in \mathbf{Z}\right\}$ with $a, b \notin \mathbf{R}^{2} \times\{0\}$. As before, $a b a^{-1}=b^{k}$ and therefore $\Gamma$ is abelian. So the elements of $\left.\mathbf{R}^{2} \times\{0\}\right) \cap \Gamma$ can be written as $a^{n} \cdot b^{-n}=\left(a \cdot b^{-1}\right)^{n}$ and this is not dense in $\mathbf{R}^{2} \times\{0\}$.

Remark. Three elements $\mathbf{u}, \mathbf{v}, \mathbf{w} \in \mathbf{R}^{3}$ can generate a dense subgroup of $\mathbf{R}^{2}$. In fact, it suffices to take $\mathbf{u}=\lambda \mathbf{v}+\mu \mathbf{w}$ with $\lambda, \mu$, and $\lambda / \mu \in \mathbf{R} \backslash \mathbf{Q}$. So, a priori, it is possible to have $\overline{h\left(\mathbf{Z}^{3}\right)}=\mathbf{R}^{2} \times\{0\}$.

Proposition 2. $\mathscr{G}_{6}$ is not realizable.

Proof. If such a realization exists, the subgroup $h\left(\mathbf{Z}^{3}\right)$ is normal in $\Gamma$. Let $h\left(\mathbf{Z}^{3}\right)=\left\langle\left(p_{1}, 0\right),\left(p_{2}, 0\right),\left(p_{3}, 0\right)\right\rangle$ and $h(\mathbf{Z})=\langle(p, t)\rangle$ with $t>0$; then the normality condition can be written as $e^{-\Lambda t} \cdot p_{i}=\sum_{j=1}^{3} \lambda_{1}^{j} \cdot p_{j}$, where $\lambda_{i}^{j} \in \mathbf{Z}$.

The matrix $A=\left(\lambda_{i}^{j}\right)$ corresponds, in fact, to $\varphi_{*}: \mathbf{Z}^{3} \rightarrow \mathbf{Z}^{3}$ so it is invertible, and, as we are assuming orientability, we have $\operatorname{det} A=1$. Let $v_{1}=\left(a_{1}, b_{1}, c_{1}\right)$ and $v_{2}=\left(a_{2}, b_{2}, c_{2}\right)$, where $p_{1}=\left(a_{1}, a_{2}\right), p_{2}=\left(b_{1}, b_{2}\right)$, and $p_{3}=\left(c_{1}, c_{2}\right)$. From the above equations we have

$$
A v_{1}=a \cdot a_{1}+a \log a \cdot v_{2}, \quad A v_{2}=a \cdot v_{2},
$$

where $a=e^{-t}$.

Completing $v_{1}, v_{2}$ to a basis $\left\{v_{1}, v_{2}, v_{3}\right\}$, the matrix $A$ can be written

$$
\left(\begin{array}{ccc}
a & 0 & \alpha \\
a \log a & a & \beta \\
0 & 0 & a^{-2}
\end{array}\right)
$$

and satisfies

$$
2 a+\frac{1}{a^{2}}=p, \quad a^{2}+\frac{2}{a}=q,
$$

with $p, q \in \mathbf{Z}, 0<a<1$, and $a \in \mathbf{R} \backslash \mathbf{Q}$. But this is impossible because the equations imply that $p a^{2}-2 q a+3=0$ and hence $a=\left(q \pm \sqrt{q^{2}-3 p}\right) / p$. In particular, $\sqrt{q^{2}-3 p} \in \mathbf{R} \backslash \mathbf{Q}$. Substituting $a$ in the first equation above we conclude, after a short computation, that $p=q=3$, which is in contradiction with $a \in \mathbf{R} \backslash \mathbf{Q}$. So $\mathscr{G}_{6}$ is not realizable.

Proposition 3. The Lie algebras of the $\mathscr{G}_{7}$ family with $k \in \mathbf{Q}$ are not realizable.

Proof. Proceeding as in Proposition 2, we obtain that $e^{-t}$ and $e^{-k t}$ are eigenvalues of $A$.

The characteristic polynomial of $A, x^{3}-p x^{2}+q x-1$, has three roots, $\xi$, $\xi^{k}$, and $\xi^{-(k+1)}$ with $\xi=e^{-t}$. As $t>0$ we have $0<\xi<1$. This implies, 
from standard arguments in Galois theory (see lemma below), that $k \notin \mathbf{Q}$; i.e. the Lie algebras of $\mathscr{G}_{7}$ with $k \in \mathbf{Q}$ are not realizable.

The authors are grateful to P. Ara for his remarks about the following lemma.

Lemma. Let $f(x)=x^{3}-p x^{2}+q x-1$ be a polynomial with $p, q \in \mathbf{Z}$. If there are $k \in \mathbf{R} \backslash\{0\}$ and $\xi \in(0,1)$ such that the roots of $f(x)$ can be written as $\boldsymbol{\xi}, \xi^{k}, \boldsymbol{\xi}^{-(k+1)}$, then $k \in \mathbf{R} \backslash \mathbf{Q}$.

Proof. First we observe that any rational root of this polynomial must be 1 or -1 , and so it is irreducible over $\mathbf{Q}$. Hence the Galois group of $f(x)$ over $\mathbf{Q}$ is $\mathbf{Z}_{3}$ or the symmetric group $S_{3}$. In both cases there is an automorphism $\sigma$ of the splitting field $\mathscr{K}$ of order 3 , which is the identity over $\mathbf{Q}$. This automorphism permutes the roots, i.e. $\sigma(\xi)=\xi^{k}, \sigma\left(\xi^{k}\right)=\xi^{-k-1}, \sigma\left(\xi^{-k-1}\right)=$ $\xi$, or $\sigma(\xi)=\xi^{-k-1}, \sigma\left(\xi^{k}\right)=\xi, \sigma\left(\xi^{-k-1}\right)=\xi^{k}$.

If $k=p / q$, using that $\sigma\left(x^{1 / q}\right)= \pm \sigma(x)^{1 / q}$, we obtain $\xi^{-k-1}=\sigma\left(\xi^{k}\right)=$ $\sigma\left(\xi^{p / q}\right)=\sigma\left(\left(\xi^{p}\right)^{1 / q}\right)=\left(\sigma(\xi)^{p}\right)^{1 / q}=\xi^{k^{2}}$ in the first case and $\xi=\sigma\left(\xi^{x}\right)=$ $\xi^{(-k-1) k}$ in the second. This implies that $k^{2}+k+1=0$, which is impossible. Thus $k \notin \mathbf{Q}$ and the lemma is proved.

Example. Now we give an example of a Lie flow on a compact manifold $M$ transversely modeled over a Lie algebra $\mathscr{G}$ of the family $\mathscr{G}_{7}$ with structural Lie algebra of dimension 2 .

Let

$$
A=\left(\begin{array}{ccc}
2 & 1 & 1 \\
1 & 1 & -1 \\
1 & 0 & 3
\end{array}\right)
$$

be an element of $\operatorname{SL}(3 ; \mathbf{R})$.

The eigenvalues are $\lambda_{j}=2+2 \cos \left(\left(6 \pi_{j}-4 \pi\right) / 9\right)$ where $j=1,2,3$. We have $2+2 \cos (8 \pi / 9)<1<2+2 \cos (14 \pi / 9)<2+2 \cos (2 \pi / 9)$. If we let $\xi=\lambda_{2}$, there is a $k<0$ such that $\xi^{k}=\lambda_{3}$. In this case $\lambda_{1}=\xi^{-k-1}$. Here $k$ is the quotient of logarithms of algebraic numbers. Notice that this is a necessary condition for the corresponding algebra to be realizable.

Thus we have the eigenvectors $u_{j}=\left(\lambda_{j}-3, \lambda_{j}\left(\lambda_{j}-3\right)-1,1\right)$.

A computation shows that the components of these vectors have irrational quotient, i.e. they induce dense linear flows in $T^{3}$.

Now we consider the compact manifold $T_{A}^{4}=T^{3} \times \mathbf{R} / \sim$, where $(x, t) \sim$ $(A \cdot x, t+1)$. As the direction given by $u_{1}$ is invariant by $A$, it induces a global flow in $T_{A}^{4}$. This flow is transversely modeled over the Lie algebra of $\mathscr{G}_{7}$ with $k=\log \lambda_{3} / \log \lambda_{2}<0$. To verify this we observe that an invariant transverse parallelism in $T^{3} \times \mathbf{R}$ is given by

$$
e_{1}=\xi^{t} u_{2}, \quad e_{2}=\xi^{k t} u_{3}, \quad e_{3}=-\frac{1}{\log \xi} \frac{\partial}{\partial t},
$$

and it satisfies $\left[e_{1}, e_{2}\right]=0,\left[e_{1}, e_{3}\right]=e_{1},\left[e_{2}, e_{3}\right]=k e_{2}$. 
Remark. We do not know any realization of $\mathscr{G}_{8}$ with $h \neq 0$ and $\operatorname{codim} \overline{\mathscr{F}}=1$. Case 4. $\operatorname{codim} \overline{\mathscr{F}}=0$.

This is a trivial case because the transverse algebra coincides with the structural algebra and so it is abelian. Only $\mathscr{G}_{1}$ is realizable (a linear dense flow on $\left.T^{4}\right)$.

\section{REFERENCES}

1. Y. Carrière, Journées sur les structures transverses. Toulouse 1982, Asterisque 116 (1984).

2. A. ElKacimi and M. Nicolau, Espaces homogènes moyennables et feuilletages de Lie, Publ. IRMA 12, Lille, 1988.

3. E. Fedida, Sur les feuilletages de Lie, C. R. Acad. Sci. Paris 277 (1971), 999-1002.

4. C. Godbillon, Feuilletages, Institut de la Recherche Mathematique Avancée, Université Louis Pasteur, Strasbourg.

5. M. Llabrés and A. Reventós, Unimodular Lie foliations, Ann. Fac. Sci. Toulouse Math. IV (5) (1988).

6. P. Molino, Géométrie globale des feuilletages riemanniens, Proc. Kon. Nederl. Akad. Ser. A1 85 (1982), 45-76.

Departament de Matemàtiques, Universitat Autònoma de Barcelona, 08193 BellaTERRA, SPAIN 